\title{
A Pharmacist-Led, Patient-Centered Program Incorporating Motivational Interviewing for Behavior Change to Improve Adherence Rates and Star Ratings in a Medicare Plan
}

\author{
Jamie Spears, PharmD; Jill Erkens, RPh; Cathi Misquitta, PharmD, MBA, BCPS; \\ Tim Cutler, PharmD, BCGP; and Marilyn Stebbins, PharmD, FCSHP
}

\begin{abstract}
INTRODUCTION: The Medicare 5-star quality rating system was designed to drive improvements in Medicare quality and to increase accountability among Medicare plans. Medicare star ratings provide significant bonuses for plans that improve medication adherence. Envolve's pharmacy division, Envolve Pharmacy Solutions, which provides services for Medicare Advantage Prescription Drug plans, developed an in-house medication therapy management (MTM) program to improve adherence rates and subsequent star ratings. As part of this program, Envolve invested in motivational interviewing (MI) as a means to improve adherence to antihypertensives, antihyperlipidemics, and antidiabetics but recognized the need for additional staff training to ensure pharmacist success with MI techniques. Thus, Envolve engaged a consultant to help train pharmacists and evaluate the program. This best practices article describes the implementation of an MI program and subsequent changes in patient adherence and star ratings.

PROGRAM DESCRIPTION: A pharmacist-led, patient-centered adherence program incorporating $\mathrm{MI}$ for behavior change was developed and implemented at Envolve. The program used didactic learning, coaching and skills assessments, and a train-the-trainer (TtT) intervention. This approach resulted in improved adherence rates in all 3 therapeutic classes immediately. In addition, a quality improvement process was incorporated to evaluate the improvements in adherence with this new program over 24 months.
\end{abstract}

OBSERVATIONS: Key findings of the program are as follows: (a) the program increased adherence rates 5-9 percentage points (chi-square tests for all plans and drug classes measured, $P<0.05$ ) over 5 years and improved Medicare star ratings by 1-2 stars; (b) there is a need for support of pharmacy MTM managers to ensure continued success of the program; and (c) there is value in a TtT program for managers that allows them to provide continuous evaluation and feedback to staff for improvement.

IMPLICATIONS: Each year, as the Medicare star ratings system matures and plans are held more accountable for improving adherence measures, high star ratings become more difficult to attain. This MI TtT program for pharmacists allows for rapid cycle change in response to these challenges.

J Manag Care Spec Pharm. 2020;26(1):35-41

Copyright $\odot 2020$, Academy of Managed Care Pharmacy. All rights reserved.

\section{What is already known about this subject}

The Centers for Medicare \& Medicaid Services (CMS) emphasize medication therapy measures, including adherence for Parts C and D plans, to achieve higher Medicare star ratings.

Health plans may be able to improve multiple star ratings and overall plan performance with strategies aimed at improving medication adherence rates.

Motivational interviewing (MI) is an evidenced-based and effective intervention for influencing patient behaviors and associated positive health outcomes.

\section{What this study adds}

Patient-centered MI techniques used in a telephonic pharmacistled intervention at the plan level can be used to improve adherence in targeted drug classes and, thus, Medicare star ratings. A train-the-trainer model in MI for pharmacy management teams can be used to promote continuous quality improvement in medication adherence and, thus, Medicare star ratings.

A 5-star quality rating system, implemented to drive improvements in Medicare quality and to increase accountability among Medicare Part D and Part C plans for the care provided to beneficiaries, was developed by the Centers for Medicare \& Medicaid Services (CMS) in 2007.' In 2011, the Affordable Care Act (ACA) strengthened the effect of the CMS star ratings by providing quality incentive payments to these plans. ${ }^{2}$ Bonus payments for improved ratings in quality measures helped plans focus efforts on strategic initiatives to improve measured areas, which include member satisfaction and clinical outcomes. The initial demonstration period (20122014) enabled plans scoring at least 3 stars to receive a bonus payment for quality. However, beginning with the 2015 star ratings (reflecting the 2013 calendar year), plans had to achieve 4 or 5 stars to receive a bonus as outlined in the ACA. ${ }^{3}$

Medicare star ratings are calculated using a weighted average of 34 Part C measures and 14 Part D measures for Medicare Advantage Prescription Drug (MAPD) plans. These measures are weighted at $1,1.5$, or 3 . Adherence to medications for the treatment of diabetes, hypertension, and hyperlipidemia are 


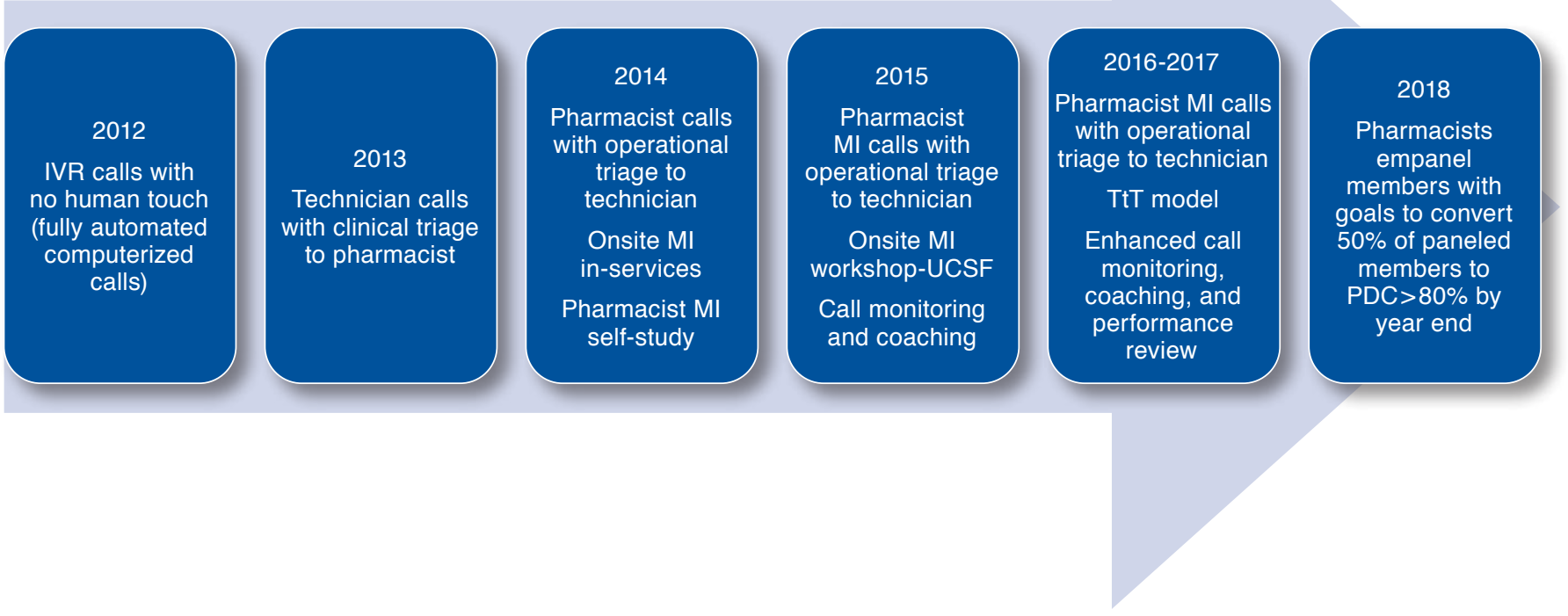

$I V R=$ interactive voice response; $M I=$ motivational interviewing; $P D C=$ proportion of days covered; $T t T=$ train-the-trainer; UCSF = University of California, San Francisco.

heavily weighted by $\mathrm{CMS},{ }^{4}$ since clinical outcomes and quality of life measures have been shown to improve with increased adherence. ${ }^{5-7}$ Adherence measures are considered intermediate outcome measures and thus receive a weighting of 3 . Consequently, they directly affect over $12 \%$ of a plan's overall star rating. In addition, several Part $C$ measures are related to the treatment of these 3 conditions; therefore, adherence to these medications could affect an additional 11\% of the overall rating. ${ }^{4}$ Considering these outcome measures and the sizable bonus plans received for obtaining at least 4 stars, it benefits plans to implement programs to improve medication adherence.

Envolve Pharmacy Solutions, a wholly owned subsidiary of Centene Corporation, is a pharmacy benefit manager with 20 years of experience that has focused on governmentsponsored health care and commercial business. Envolve has supported MAPD plans since the implementation of Medicare Part D in 2006 and has an in-house pharmacy-run medication therapy management (MTM) program providing MTM and adherence programs for its members. In 2012, Envolve had roughly 225,000 MAPD members, 24\% of whom were nonadherent in the star metrics. Pharmacy and quality teams evaluated star measures and their effect on members and the plan. They determined that the star ratings were not optimal (ranging from 2.5-5, depending on the plan and the measure). Consequently, an adherence program was developed with a goal of improving star ratings. This best practices article follows Evolve's adherence program improvements between 2013 and 2018 for 4 MAPD Envolve health plans.

The Envolve adherence program evolved over time (Figure 1). The initial adherence program that was implemented used an interactive voice response (IVR) call that went to members who were nonadherent to statins, oral diabetes medications, and renin-angiotensin system antagonists (RASAs). Nonadherence was defined as proportion of days covered (PDC) $<80 \%$. During the IVR call, members were asked a series of barrier analysis questions. Based on the member's response, a possible, prerecorded solution was provided. A 3-month evaluation revealed no improvements in adherence. As a result, the IVR calls were stopped in 2013, and pharmacy technicians conducted real-time, telephonic outreach to members identified as nonadherent. The technicians asked members a similar list of barrier analysis questions. They also facilitated switches to 90-day supplies and offered refill synchronization. When appropriate, adherence calls were transferred to pharmacists 
for management of clinical issues. By late 2014, the technicianrun adherence program demonstrated modest increases in adherence rates but did not reach the targeted 4-star threshold.

It was noted that few calls were transferred to pharmacists. The pharmacy management team concluded that future adherence programs needed to address member engagement and behavior change towards adherence to avoid continual reidentification of the same nonadherent members. A pharmacist-led adherence program based on patient-centered motivational interviewing (MI) for behavior change was then selected for the in-house, telephonic adherence MTM program because MI has been shown to change patient behavior in several settings. ${ }^{8-11}$ At the time of this intervention, few pharmacists had been trained in this approach and no evidence of MI techniques being used to improve adherence in the setting of CMS star ratings and in-house MTM programs was found in the literature.

\section{Program Description}

The pharmacist-led, patient-centered adherence program incorporating MI for behavior change was not initially designed as a multiyear continuous quality improvement program. However, the management team recognized that improvements in adherence would take time and commitment to continuous quality improvement and rapid cycle change. The initial step was to provide the pharmacists knowledge-based training on MI. At this time, there were 9 pharmacists involved in the adherence program, serving a Medicare population of 315,539. Pharmacists were required to attend 2 onsite in-services on MI, and Envolve provided reference material on MI for pharmacist self-study. ${ }^{12}$ During this initial phase, the pharmacy management team reported that the pharmacists continued to rely on their standard consultation skills when talking to members, and adherence rates did not improve by the end of 2014 (Figure 2). Although the management team believed that incorporating MI techniques with members identified as nonadherent could improve adherence and star ratings, they were not experts in the use of MI. This led to eliciting assistance from external experts in the field.

A business case was presented to upper management to secure funding for an outside consultant to provide skillsbased MI and application training. The pharmacy management team researched MI training programs and contacted 4 California schools of pharmacy to determine expertise in MI and ability to develop a training program tailored to Envolve's adherence program. Subsequently, a consulting agreement was signed with the University of California, San Francisco (UCSF).

\section{Year 1 (2015)}

The Envolve pharmacy management team met with the UCSF pharmacist consultants to develop a stepwise program that included knowledge, comprehension, and application of MI training. Because the Envolve pharmacists already had initial training in MI, during the first phase the UCSF pharmacists listened anonymously to 5 live adherence calls per pharmacist. This demonstrated whether MI skills, such as open-ended questions, affirmations, reflective listening, and summaries were used and provided an opportunity to create a tailored curriculum for pharmacist-specific process improvement. The curriculum included (a) a pharmacist self-assessment, (b) training on individualized scripted introductions, (c) feedback on MI skills and techniques, (d) role-playing, and (e) an opportunity for peer review. In addition, individual coaching sessions ranging from 30-60 minutes were provided for each pharmacist. Systems issues that prevented effective integration of MI during adherence calls were identified and discussed with plan leadership.

As a result of the training and pharmacist feedback, 2 areas were initially targeted for continuous quality improvement: personalized introductory scripts for the pharmacists and identification of a patient-specific adherence barrier to promote member activation, engagement, and patient-guided problem solving. The UCSF consultants interacted with the Envolve team from February 2015 through April 2015.

The Envolve pharmacy management team realized that it would take time for the pharmacists to develop the targeted MI skills and that they had to establish a process to continuously evaluate the pharmacists' MI skills once the UCSF consultants left. The first process implemented was a peer-review model. Pharmacists were assigned to anonymously listen to another pharmacist's live call and to provide an assessment using an MI assessment form developed by the UCSF consultants. The form provided evaluation of call structure and use of MI techniques to promote a behavior change. The assessment was divided into 2 phases over 4 months as follows:

- Phase 1: evaluate the pharmacist's call structure (introduction, purpose of the call, and permission to conduct the call) and use of open-ended questions.

- Phase 2: evaluate advanced MI techniques, such as reflective listening and evocation, to develop a patient-centered goal and plan through shared decision making.

This process was initially effective for improving pharmacist call structure; however, it was not an objective process for feedback on MI techniques due to variations in MI proficiency among the pharmacists performing the evaluation. Also, listening to live calls produced delayed and incomplete assessments, rendering feedback only mildly effective. In most of the MAPD plans supported by Envolve, adherence either remained unchanged in 2015 or showed minor improvements (Figure 2).

\section{Year 2 (2016)}

Because the peer review process had only moderate success in changing pharmacist behavior, Envolve engaged the UCSF consultants for a second year to resolve shortcomings. In year 2, a 

2013-2018 Across 4 Medicare Advantage Prescription Drug Plans ${ }^{a, b}$

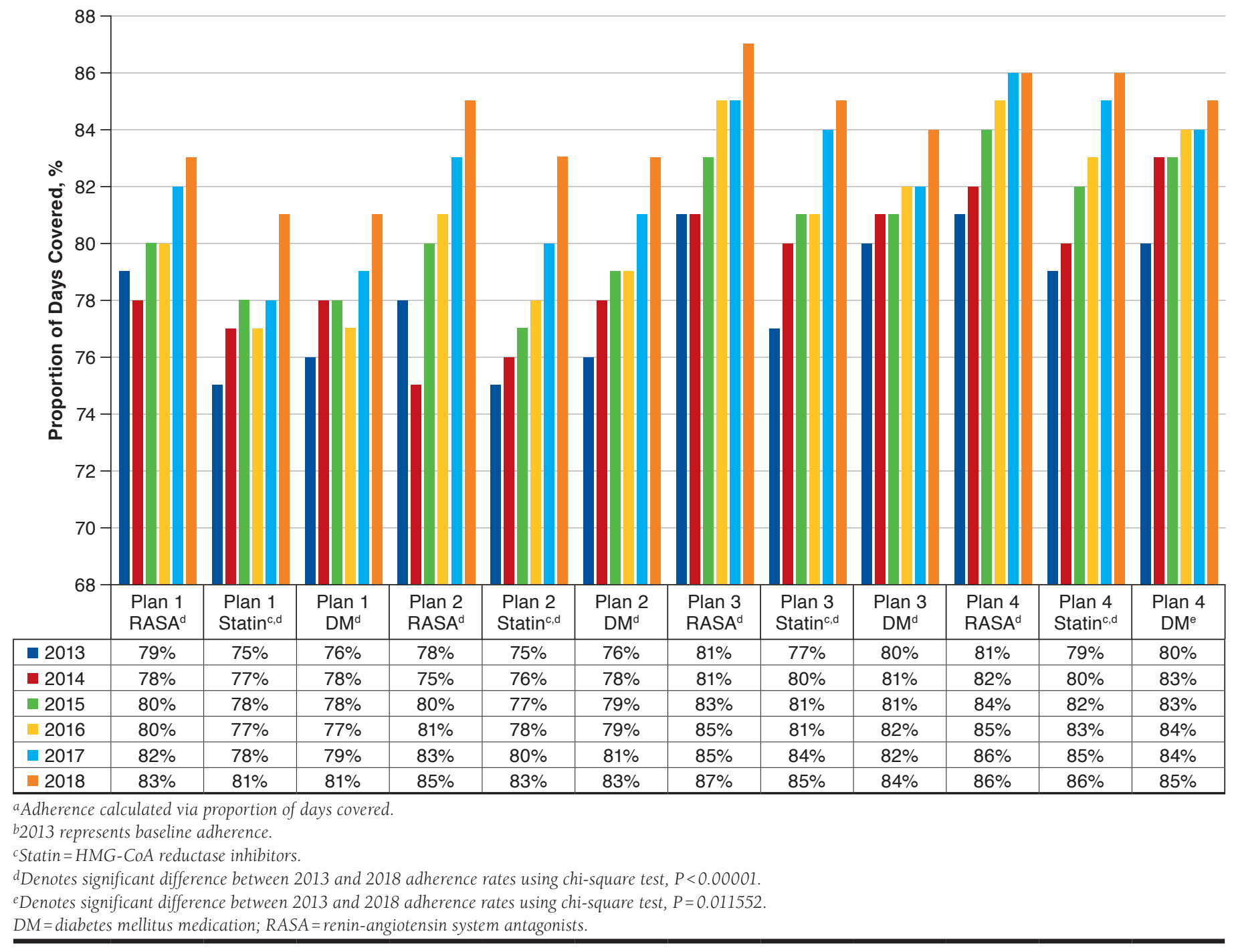

train-the-trainer $(\mathrm{Tt} \mathrm{T})$ program with the managers was included so that MI evaluations and coaching could continue when the consultants left. Focus groups with 2 independent facilitators were conducted with the pharmacists to determine issues that they were having with their MI skills and to gather individual pharmacist feedback on knowledge, comprehension, and attitudes toward MI. The TtT program focused on 2 areas identified in these focus groups: manager evaluation of adherence calls using a standardized rubric and pharmacist coaching.

For the TtT program, UCSF consultants worked with the pharmacy management team to use a new evaluation tool and rubric to objectively assess pharmacist effectiveness on the calls. The rubric was based on the following global concepts of MI: empathy, collaboration, autonomy and support, direction, and evocation. ${ }^{13}$ Behavior counts on how often open-ended questions and reflections were used by the pharmacists were also assessed. Scoring ranged from a low MI score of 1 to a high MI score of 5 . A score of 1 indicated that the pharmacist was antagonist or argumentative with the member. Conversely, a score of 5 meant that the pharmacist demonstrated exemplary MI skill during the conversation with the patient. The consultants and manager independently evaluated 3 calls per pharmacist and met to compare and discuss the evaluations to create 1 evaluation. This process helped the manager develop a consistent scoring system using the rubric. The consultant also attended the 
manager-pharmacist feedback and coaching sessions to provide evaluation and feedback on coaching skills with each pharmacist. Finally, the consultants evaluated 3 calls per pharmacist to specifically determine if they were reaching the targets set the previous year. The pharmacist introductions were deemed effective, and patient adherence barriers were readily identified, which appeared to lead to improvements in member engagement. This process was completed over 2 months.

Based on the focus group outcomes and individual call evaluations, it was determined that pharmacists struggled to identify opportunities for setting medication adherence goals with the member, which led to a lack of follow-up with patients. Accordingly, the consultants, managers, and pharmacists developed an MI skill development template that focuses on the MI skill needed to demonstrate the global MI concept. For example, the use of open-ended questions instead of closedended questions shows strength in collaboration. Pharmacists set individual goals for MI score improvement using the MI skill development template as part of their annual selfevaluations and established performance improvement plans when necessary to address deficiencies. These self-evaluations became part of their annual review. The consultants spent 2 months in 2016 with the Envolve pharmacists; however, the Envolve managers continued to provide ongoing evaluation and coaching throughout 2016.

Given the systems challenges with call assessments, as well as inconsistencies with rubric scoring, it was decided to manually record calls for evaluation (rather than listen to live calls) and to transition call evaluation to a single manager. Calls were audited and scored based on the same 5-point scoring rubric focused on the global concepts of MI. The individual pharmacist met with a manager to listen to the recorded call and received written and verbal coaching based on MI proficiencies. The goal for each pharmacist was to reach a 4 or higher by November 2016 when averaging all 5 of the global MI concepts.

Limitations still existed with the manually recorded calls. There were few calls available to evaluate, since not all of the pharmacists' calls were recorded, and it was difficult for the scoring manager to mine for calls appropriate for evaluation. Consequently, feedback to pharmacists was delayed and less frequent than desired. Despite these limitations, the pharmacists were able to achieve the scoring goal, and the objective call evaluation process and feedback model validated MI proficiency for the team. In addition, the plans' adherence rates improved for the 3 targeted drug classes (Figure 2), leading to a CMS 4-star rating for those Part D measures.

\section{Year 3 (2017)}

During the third year of this program, the Envolve Medicare MTM program was implemented across the entire enterprise, resulting in significant staff growth. To support the current managers and continue to innovate and improve, Envolve hired another manager, as well as a director for the MTM program. The director was responsible for developing a calibration team to address the need for an increased number of calls to be monitored as a result of pharmacist staff growing by a factor of 3. The calibration team met monthly to listen to 1 call, score that call, and then discuss evaluations to promote consistency and scoring alignment. Each manager was then tasked with evaluating the calls for pharmacists reporting to them. The UCSF consultants were invited back for a final visit to review the progress made, evaluate 2 calls per pharmacist based on the rubric developed in year 2 , and continue to provide feedback to the managers.

During this visit, the consultants and the calibration team simplified the rubric to provide just 2-3 examples for MI core behaviors rated on a scale of $1-5$, since the team felt it made scoring more consistent after the team felt some of the examples were duplicative. By the end of 2017, 88\% of staff pharmacists were able to maintain an average MI score of 4 or greater compared with $84 \%$ at the beginning of the year. Performance improvement plans were successfully executed where necessary. Moreover, adherence rates for the 3 targeted drug classes improved by $1-2$ percentage points over the previous year (Figure 2).

Currently, the process involves a robust training plan, a call monitoring program, and a performance improvement plan. The MTM staff consists of 56 employees who serve over 240,000 members. An automated system records all pharmacist calls so that they are available in real-time for scoring. Call monitoring/scoring has transitioned to an MTM technician with a background in customer service call audits. The technician, along with the managers and program director, meet monthly for calibration calls to ensure that each pharmacist is scored and coached in a consistent manner. Newly hired pharmacists are introduced to MI with on-demand webinar training. ${ }^{14}$ The management staff reviews MI techniques and conducts role play sessions specific to Envolve's call program. This approach is based on the UCSF consulting program, as well as materials from other MI experts. ${ }^{12,14}$

Pharmacists attend a call review session for baseline scoring with their managers and the director within the first 90 days of training. If the pharmacist scores a 4 or better upon baseline training, they are moved to quarterly call monitoring. If pharmacists do not reach the expected goal, they are coached and monitored monthly until improvement is demonstrated or a performance improvement plan is developed.

\section{Observations}

It is difficult to improve and then maintain medication adherence rates and, thus, Medicare star ratings, without novel, 
continuous, and real-time interventions. Plans must be willing to take a chance, fail fast, pivot, and move on to new approaches when adherence and ratings do not improve.

Although the initial course of action in this program was to train pharmacists in MI, using a school of pharmacy consultant, a need for a longer-term strategy to continuously evaluate and maintain pharmacists' skills was identified. A TtT program provided in-house managers with the skills to continuously monitor, evaluate, innovate, and implement programs to help pharmacists engage patients, with a goal of improving adherence rates in real time without the need for long-term consultants. This program successfully improved adherence rates for the 3 targeted drug classes-antihypertensives 6.8\%, antihyperlipidemics $8.5 \%$, and antidiabetics $5.8 \%$-on average over the baseline star rating (Figure 2). Of note, however, plans need to continually evaluate and support their management staff. In this experience, the addition of a director to evaluate and support the frontline managers was key to the success of the program.

\section{Implications}

The 2019 CMS star rating targets for adherence measures (representing work completed in 2017) are several percentage points higher than the previous year, suggesting a need to continually improve and evolve the adherence program. This study demonstrates that an adherence program that includes rigorous training in MI and continuous quality improvement can positively affect adherence rates and CMS star ratings. Furthermore, health plans dedicated to providing targeted and ongoing coaching to MTM staff can further improve adherence rates over time. While programs that include MI training alone may improve adherence rates, this study indicates that additional improvements can be achieved if MTM staff have individual skill development plans that are continuously evaluated, acted upon, and updated.

\section{Authors}

JAMIE SPEARS, PharmD; JILL ERKENS, RPh; and CATHI MISQUITTA, PharmD, MBA, BCPS, Envolve Pharmacy Solutions, Orlando, Florida. TIM CUTLER, PharmD, BCGP, Department of Pharmacy, University of California, Davis, Medical Center and School of Pharmacy, University of California, San Francisco, and MARILYN STEBBINS, PharmD, FCSHP, School of Pharmacy, University of California, San Francisco.

AUTHOR CORRESPONDENCE: Marilyn Stebbins, PharmD, FCSHP, Professor of Clinical Pharmacy, Vice Chair of Clinical Innovation, UCSF School of Pharmacy, 533 Parnassus Ave., Box 0622, Rm. U585, San Francisco CA 94143. Tel.: 415.476.3955; E-mail: Marilyn.stebbins@ucsf.edu.

\section{DISCLOSURES}

Funding was provided by Envolve Pharmacy Solutions, which contracted with the University of California, San Francisco (UCSF), School of Pharmacy for the development and implementation of the motivational interviewing and train-the-trainer programs described in this best practices article Spears, Erkens, and Misquitta are employees of Envolve Pharmacy Solutions. Stebbins and Cutler are faculty in the Department of Clinical Pharmacy at the UCSF School of Pharmacy, who were contracted through Envolve Pharmacy Solutions to provide consulting services for this best practice.

\section{ACKNOWLEDGMENTS}

The authors thank the following individuals from Envolve Pharmacy Solutions for their participation in the development and coaching for this best practice: Heather P. Gregory, BCGP, RPh, Manager, Clinical Pharmacy Operations (MTM); Carrie Holden, PharmD, Manager, Clinical Pharmacy Operations (MTM); and Jeffrey R. Stansberry, PharmD, Manager, Clinical Pharmacy Operations (MTM). The authors also thank Judie Tran, PharmD, UCSF School of Pharmacy, and Vinh Nguyen, PharmD, Kaiser Permanente, Oakland, for their services as focus group facilitators while they were pharmacy practice residents at UCSF School of Pharmacy and UC Davis Medical Center, respectively.

\section{REFERENCES}

1. Centers for Medicare \& Medicaid Services. Five-star quality rating system. October 7, 2019. Available at: https://www.cms.gov/Medicare/ProviderEnrollment-and-Certification/CertificationandComplianc/FSQRS.html. Accessed November 30, 2019.

2. Centers for Medicare \& Medicaid Services. The Affordable Care Act: strengthening Medicare in 2011. Available at: https://www.cms.gov/apps/ files/medicarereport2011.pdf. Accessed November 30, 2019.

3. Galewitz P. Medicare plans score higher ratings and millions in bonuses Kaiser Health News. March 7, 2016. Available at: https://khn.org/news/ medicare-plans-score-higher-ratings-and-millions-in-bonuses/. Accessed November 30, 2019

4. Centers for Medicare \& Medicaid Services. 2018 star measures and display measures (ZIP). 2018 Part C \& D star rating technical notes. Available at: https://www.cms.gov/Medicare/Prescription-Drug-Coverage/Prescription DrugCovGenIn/PerformanceData.html. Accessed November 30, 2019.

5. Ho PM, Rumsfeld JS, Masoudi FA, et al. Effect of medication nonadherence on hospitalization and mortality among patients with diabetes mellitus. Arch Intern Med. 2006;166(17):1836-41.

6. Osterberg L, Blaschke T. Adherence to medication. N Engl J Med. 2005;353(5):487-97

7. Roebuck MC, Liberman JN, Gemmill-Toyama M, Brennan YA. Medication adherence leads to lower health care costs despite increased drug spending. Health Aff (Millwood). 2011;30(1):91-99.

8. Miller WR, Sovereign RG, Krege B. Motivational interviewing with problem drinkers: II. The drinker's check-up as a preventive intervention. Behav Cogn Psychother. 1988;16(4):251-68.

9. Pringle JL, Boyer A, Conklin MH, McCullough JX, Aldridge A. The Pennsylvania Project: pharmacist intervention improved medication adherence and reduced health care costs. Health Aff (Millwood). 2014;33(8):1444-52.

10. Abughosh S, Wang X, Serna O, et al. A motivational interviewing intervention by pharmacy students to improve medication adherence. J Manag Care Spec Pharm. 2017;23(5):549-60. Available at: https://www.jmcp.org/ doi/10.18553/jmcp.2017.23.5.549.

11. Berger BA, Bertram CT. Motivational interviewing and specialty pharmacy. J Manag Care Spec Pharm. 2015;21(1):13-17. Available at: https://www. jmcp.org/doi/10.18553/jmcp.2015.21.1.13. 
A Pharmacist-Led, Patient-Centered Program Incorporating Motivational Interviewing

for Behavior Change to Improve Adherence Rates and Star Ratings in a Medicare Plan

12. Berger BA, Villaume WA. Motivational Interviewing for Health Care Professionals: A Sensible Approach. Washington, DC: APhA Press; 2013.

13. Center on Alcoholism, Substance Abuse and Addictions (CASAA), University of New Mexico. Coding instruments. MITI 3.1.1. Available at: https://casaa.unm.edu/codinginst.html. Accessed November 30, 2019.
14. HealthSciences Institute. Motivational interviewing training for health care. 2019. Available at: https://healthsciences.org/MotivationalInterviewing-Health-Coach-Training. Accessed November 30, 2019. 